\title{
Demanda de contenidos mediáticos entre adultos jóvenes de Huancayo
}

\author{
Demand for media content among young adults \\ in Huancayo
}

Ivo Eduardo Yance Carlos ${ }^{1 *}$, José María Matas Castañeda ${ }^{2}$

${ }^{1}$ Facultad de Humanidades, Universidad Continental, Huancayo ${ }^{2}$ Universidad Ramon Llull, Barcelona

\section{RESUMEN}

El panorama mediático resulta desafiante, confuso e incierto. El consumo de medios ha cambiado dramáticamente en las últimas décadas y tiene como principales factores de transformación: la distribución digital, audiencias cada vez más atomizadas, consumo bajo demanda, mayor acceso a plataformas de generación de contenidos y tecnología sin cables (Wireless Fidelity). En el esfuerzo por comprender este contexto, la presente investigación aborda la demanda de contenidos de adultos de 25 a 35 años de Huancayo, para ello se realizaron dos grupos de enfoque, uno de varones y otro de mujeres con ocho integrantes cada uno, y una encuesta (dirigida a 390 personas) para explorar las necesidades de este grupo objetivo, información que fue contrastada con la oferta presentada por los principales medios impresos (Correo y Primicia), radiales (radio Huancayo y 15:50) y televisivos (Kdena 15 y Unitel). Los resultados muestran que los medios satisfacen parcialmente las demandas del público analizado que requiere nuevos temas como, medio ambiente, educación y familia entre otros. Pero, sobre todo, la investigación revela nuevos hábitos de consumo y presencias de ofertas como Netflix, alta penetración de la televisión por cable (más del $50 \%$ ) y otros que advierten un nuevo escenario para el desarrollo de medios de comunicación y propuestas de contenido para la población huancaína.

Palabras clave: Medios de comunicación de masas, marketing y demanda.

\begin{abstract}
The panorama mediatic is challenging, confusing and uncertain. Media consumption has changed dramatically in recent decades and has as main transformation factors: digital distribution, increasingly atomized audiences, consumption on demand, greater access to content generation platfforms and Wireless technology (Wireless Fidelity). In the effort to understand this context, the present research addresses the demand for content of adults from 25 to 35 years of age in Huancayo, for which two focus groups were made, one for men and one for women with eight members each, and a survey (directed to 390 people) to explore the needs of this target group, information that was constrasted with the offer presented by the man print media ( Correo y Primicia), radio (radio Huancayo and 15:50) and television (Kdena 15 and Unitel). The results show that the media partially satisfy the demands of the analyzed public that requires new topics such as: environment, education and family among others. But, above all, the research reveals new consumption habits and presences of offers such as Netflix, high penetration of TV cable (more than $50 \%$ ) and others that warn a new scenario for the development of media and content proposals for the Huancaina population
\end{abstract}

Keywords: Mass media, marketing and demand.

Historial del artículo:

Recibido, 22 de noviembre de 2016; aceptado, 15 de diciembre de 2016; disponible en línea, 25 de junio de 2017

${ }^{*}$ Lic. en Ciencias de la Comunicación, docente de la Universidad Continental.

Correo: iyance@continental.edu.pe 


\section{INTRODUCCIÓN}

Los medios de comunicación de masas se han definido como industrias complejas, formales, que tienen fines lucrativos, por ende, operan con grandes presupuestos, son altamente competitivas y transmiten mensajes hacia un público numeroso, heterogéneo y disperso (Dominick, 2006). Dentro de los principales medios de comunicación de masas se encuentran: periódicos, libros, revistas, discos, radio, tv e internet (Biagi, 2009).

Los medios de comunicación tienen una trascendencia social importantísima pues como señala Jensen (2014), proporcionan representaciones e ideas sobre la realidad, más enfáticamente, Baran e Hidalgo (2005) manifiestan que la comunicación masiva es el proceso por el cual se crean significados compartidos entre medios y sus públicos, mientras que Biagi, afirma que los medios reflejan y afectan la política, la sociedad y la cultura.

Es así que, los medios son factores de influencia relevantes, para la presente investigación se define a los mismos como organizaciones que transmiten mensajes públicos. Estos medios desde finales del siglo anterior enfrentan diversos retos como la convergencia empresarial, distribución digital de contenido por internet y consumo bajo demanda.

Sobre la convergencia empresarial, como integración o adquisición de empresas para formar conglomerados, en el Perú, el caso más notorio es la compra por parte del grupo El Comercio (EC) de la cadena de diarios de Epensa el 2013, con esto tiene alrededor del 80 $\%$ de participación en la prensa escrita, además de contar con acciones en América TV, Canal N y Plural TV (Carrillo, 2015).

En tanto, la desintermediación, que implica el acceso directo a un producto o servicio es posible a través de internet y ello ha cambiado radicalmente las reglas de juego en el panorama de medios, además de producir la convergencia mediática, puesto que la radio, diarios y televisión se distribuyen también a través de la red.

Esto último ha impulsado el consumo bajo demanda (on demand, en inglés), puesto que el lector, televidente o usuario de medios puede acceder al contenido e información en el momento en que prefiera, esto es una gran transformación debido a que, en el modelo anterior, los medios imponían la programación, ahora es el usuario quien tiene el control; Netflix es un claro ejemplo de ello.

Y todo esto ha desencadenado cambios más radicales como la desaparición o diminución de lo masivo, Jeff Jarvis citando al sociólogo, Raymond Williams, señala que las masas han muerto y en la actualidad existen pequeños colectivos con intereses específicos (Jarvis, 2010).

Si bien hay coincidencias con la afirmación de Jarvis y Williams, resulta evidente que aún hay concentración de audiencias masivas en momentos clave como sucesos noticiosos trascendentes, encuentros deportivos diversos o acontecimientos de interés colectivo, pero en general el consumo ha cambiado sin duda alguna. Esto último por las redes sociales, mayor acceso a teléfonos móviles, variada y extensa oferta por la red, surgimiento de Google, en 1998, que entre otros factores dinamizan el mercado de medios y hacen que se requiera analizar mejor la demanda de contenidos y la oferta.

Precisamente, el marketing se encuentra centrado en la satisfacción de las necesidades y deseos del mercado, además de advertir espacios desatendidos para desarrollar propuestas de valor que satisfagan esas carencias; para el marketing los clientes son el origen y fin de los negocios (Montesinos, 2008).

Esta herramienta emplea técnicas como la investigación para detectar demandas de productos o servicios, identifica segmentos y crea conceptos que, comunicados de forma coherente y sostenida, son interiorizados por los clientes objetivo al fijarse en sus mentes, a esto se denomina posicionamiento.

Partir desde las necesidades del cliente parece algo tan obvio que se da por sentado que se atiende a un público pero sin saber exactamente quiénes son y qué requieren; dentro del desarrollo del marketing se pasó de filosofías de dirección que abordaban con mayor énfasis la producción, producto, ventas, marketing en sí, y marketing social (Araujo y Mayorga, 2002), en algunos mercados aún se mantienen los tres primeros enfoques, pero es claro que hay grandes esfuerzos por aplicar marketing en todo tipo de organizaciones.

Puesto que la base de todo desarrollo empresarial y económico se concentra en atender necesidades insatisfechas, pero íqué es una necesidad?, para Kotler y Armstrong (2013) ésta representa un estado de carencia percibida, en tanto que los deseos son formas específicas de satisfacer esas necesidades -moldeadas por la cultura, personalidad y entorno-, mientras que la demanda, está ligada a un deseo sumado a la capacidad adquisitiva, es decir a poder pagar por aquel bien o servicio.

Aquí, cabe aclarar que los medios, salvo diarios, televisión por cable y plataformas como Netflix- este último requiere de conexión a internet-, se entregan de forma libre al público, basta con tener un receptor para captar los canales de señal abierta o sintonizar emisoras radiales, pero el modelo se sostiene a través de la publicidad, venden audiencias a empresas 
interesadas en llegar al público objetivo del programa, emisora, canal o página web.

Esta es una particularidad de la industria de medios, pues tienen consumidores o usuarios a quienes se dirigen sus contenidos (programas noticiosos, de concurso, entretenimiento o páginas de deportes, información policial entre otras) y clientes-anunciantes interesados en que sus mensajes sean atendidos por estos públicos.

Entonces para este trabajo, se concibe al marketing como la disciplina orientada a identificar y satisfacer las necesidades de los clientes, en tanto que la demanda es la expresión de un contenido (programa radial, televisivo, tema de interés) específico requerido por un determinado grupo de consumidores, éstos últimos pueden ser lectores, oyentes, televidentes o cibernautas, quiénes están dispuestos a pagar por el contenido o prestar atención, interés o tiempo al consumo del mismo.

El 27 de febrero de 2015, muchos peruanos protestaron, en diversas partes del país-incluido Huancayo-, contra los programas de concurso (Esto es Guerra y Combate) y farándula (Amor, Amor, Amor) que ofertan los principales medios televisivos de señal abierta.

Los manifestantes reclamaron mayor calidad en los contenidos, respeto al horario de protección al menor y mayor cultura (Espinoza, 2015). Esta movilización inició la presente investigación al plantear la interrogante sobre la oferta brindada por la televisión y otros medios de comunicación.

Con esta premisa, se planteó la posibilidad de indagar sobre el contenido demandado por estas audiencias, dado que se encontraban y mostraban insatisfechas con las propuestas de aquel momento.

Entonces, se esbozó la siguiente pregunta de investigación żqué contenidos demandan los adultos jóvenes entre 25 y 35 años de Huancayo?, teniendo como objetivo principal, analizar la demanda de medios de comunicación entre la población objetivo en la ciudad de Huancayo.

\section{MATERIAL Y MÉTODOS}

Huancayo, provincia capital de la región Junín, se ubica a 3271 m s.n.m. y divide geográficamente en 28 distritos, de los cuales Huancayo, El Tambo y Chilca concentran el $72 \%$ de la población (364 mil 10 habitantes).

En relación a medios, cuenta con dos diarios locales, Correo y Primicia, el primero circula desde el 23 de noviembre de 1962 y el segundo a partir del 20 de julio de 1996. Correo para el año 2015, publicaba ediciones diarias de 32 páginas, en tanto que Primicia, edita, con similar periodicidad, 24 páginas.

Para Alfaro (1997), partir por el público es una verdad incuestionable en comunicación, no obstante, esta afirmación para ciertos medios implica exclusivamente medir sintonía, lectoría, rating o tráfico (visitas a una página web), pero no hay una real búsqueda de los intereses de las audiencias en profundidad.

Por ello, en este trabajo se planteó conocer hábitos de consumo de medios e intereses temáticos a través de una investigación de mercados, herramienta que une como un puente al vendedor con el consumidor (García, 2010), mediante dos grupos de enfoque (focus group en inglés), uno de varones y otro de mujeres con ocho integrantes cada uno y una encuesta aplicada a 390 personas.

En la primera parte exploratoria, con los grupos de enfoque, se preparó una guía semiestructurada para dejar libremente a las y los participantes expresar sus opiniones y sensaciones sobre los medios de comunicación, la riqueza de la técnica se mostró al recoger versiones sobre el uso, consumo y percepciones de diarios, emisoras, canales e incluso nuevos medios, como veremos más adelante.

Tras la realización de los grupos de enfoque, que se dieron en un lugar céntrico y fueron grabados en audio y vídeo para luego evaluar y sistematizar las respuestas, se emplearon algunas de las ideas surgidas en los mismos para perfilar mejor la encuesta.

El cuestionario consideró interrogantes sobre consumo, frecuencia, horarios de sintonía y especialmente el interés de contenidos por parte de la población objetivo -pobladores entre 25 y 35 años-, para el trabajo de campo, realizado entre el 16 de noviembre y 12 de diciembre de 2015, se contactó a estudiantes universitarios quienes encuestaron en zonas de alto tránsito peatonal en Huancayo, El Tambo y Chilca, en total se aplicaron 390 encuestas válidas, la muestra se calculó probabilísticamente, mediante el uso de la fórmula de población finita con un porcentaje de confianza del $95 \%$.

Tabla 1. Población y muestra del estudio.

\begin{tabular}{lcccc}
\hline Distrito & Población & $\begin{array}{c}\text { \% de la } \\
\text { población }\end{array}$ & $\begin{array}{c}\text { \% de la } \\
\text { muestra }\end{array}$ & Muestra \\
\hline El Tambo & 161,429 & 44 & 44 & 172 \\
Huancayo & 116,953 & 32 & 32 & 124 \\
Chilca & 85,628 & 24 & 24 & 94 \\
\hline
\end{tabular}

Margen de error: $\pm 0.05 \%$, asumiendo la máxima dispersión en los resultados $(p=q=0.5)$ y una confiabilidad del $95 \%$.

Forma de muestreo: Entrevista personal con trabajo de campo realizado a pie de calle en los principales puntos de circulación de personas de cada distrito. 


\section{RESULTADOS}

A la pregunta de ¿̇cuál es el tema de mayor interés? La figura 1, muestra la preferencia sobre temas ligados a medio ambiente, medicina-salud, familia-educación, tecnología y ciencia, mientras que los de menor interés son política, deporte o entretenimiento.

En relación al grupo de enfoque resulta valioso destacar que los participantes dudan de las noticias propaladas por los medios, tienen cierta desconfianza, pues afirman que algunos conductores de espacios tienen intereses políticos, asimismo manifiestan que desean saber sobre deportes distintos del fútbol, más sobre historia, testimonios positivos de emprendedores y personas que salieron adelante, así como conocer de dónde son los productos que se consumen localmente. En tanto que, en hábitos de consumo de diarios, radio y televisión, las preferencias fueron muy variadas, los de la red, la audiencia está distribuida de la siguiente forma: Correo (18,4 \%); El Comercio (8,7 \%); Gestión (2,3\%); La República (1,8 \%).

Sobre la preferencia por emisoras radiales (figura 3) RPP tiene (17,6 \%), Radio Huancayo (15,4 \%), Nueva $Q(10,1 \%)$ y $15: 50(8,7 \%) ;$ Karibeña $(7,9 \%)$, en esta pregunta se halló un número elevado de consumo de otras señales $(18,1 \%)$, entre este grupo figuran Oxígeno, Studio 92, La Zona y Antena Sur.

Mientras que en el caso de televisión se consultó sobre la tenencia de TV-cable $(50,4 \%)$ cuenta con este servicio, entre ellos prefieren las señales de: Fox, CMD, Canal $\mathrm{N}$ y Plus TV, en tanto que entre los que no cuentan con este servicio la audiencia se distribuye entre América TV (41,6\%); Kdena 15 (10,2 \%); Panamericana Tv (8,5 $\%)$; Unitel $(6,5 \%)$; Canal $21(2,4 \%)$ y un $(21,5 \%)$ no vio nada (figura 4 ).

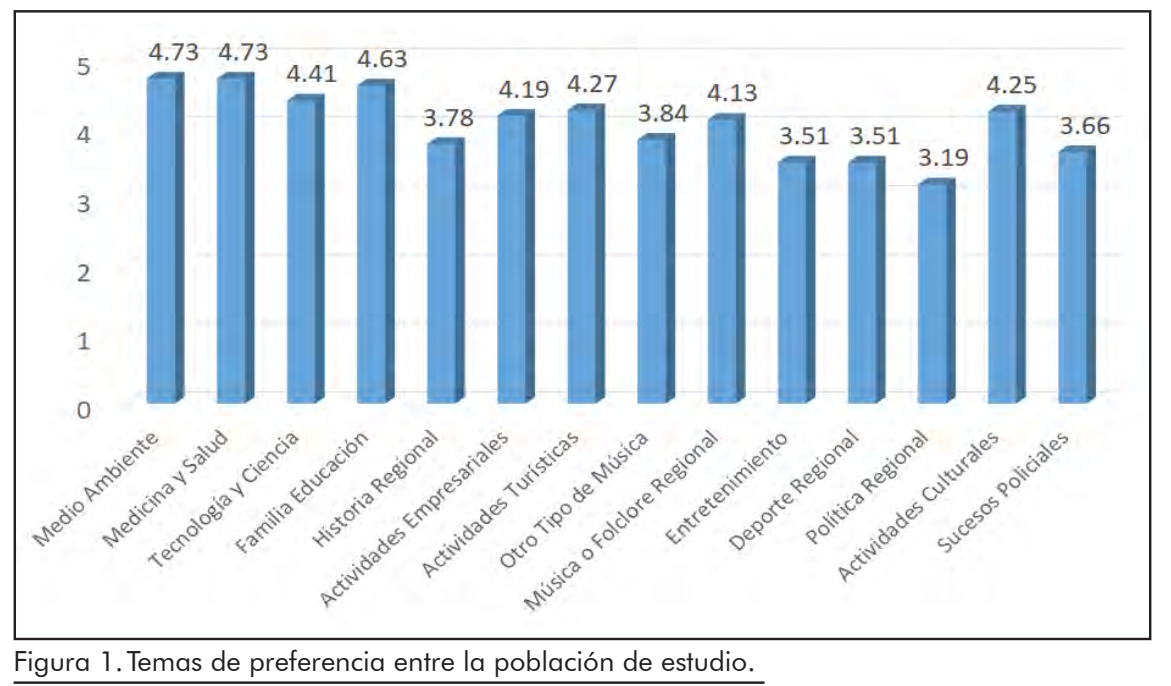

periódicos se leen preferentemente por las mañanas (70\%), mientras que la sintonía radial de lunes a viernes se concentra en las primeras horas del día, entre 6 y 9 de la mañana, hay $38 \%$ de oyentes, mientras que los fines de semana la radio tiene una audiencia que se extiende de siete de la mañana hasta mediodía (49 $\%)$, en cuanto a la televisión, el horario estelar (prime time), entre siete y once de la noche, presenta el $45 \%$ de audiencia, es oportuno destacar que de 6 a 9 de la mañana hay un grupo considerable de 19,7\%.

Sobre la preferencia de diarios impresos el grupo de estudio mostró clara preferencia por Correo $(63,9 \%)$, seguido muy de lejos por Perú 21 (5,4\%), Trome (4,9 $\%)$ y el Comercio $(4,3 \%)$, Primicia que es el otro diario local tiene un escaso $(0,5 \%)$, la figura 2 , pero también analizamos el consumo de diarios digitales más de la mitad de los encuestados indicó que no lee en digital $(52,4 \%)$ y entre quienes si consumen prensa a través
Además, en el estudio se indagó sobre el seguimiento de información de Huancayo o la región a través de internet, un $(56,7 \%)$, manifestó que no sigue nunca información de este tipo, en cuanto a la tenencia de teléfonos inteligentes (Smartphones) y conexión a la red $(60,8 \%)$ tiene esta opción, precisamente este dispositivo (móvil) es el preferido para conectarse a Internet, siguen el ordenador de escritorio, las computadoras portátiles y las tabletas, en orden de importancia, en cuanto a la plataforma de entretenimiento Netflix, $(1,8$ $\%)$ es usuario de este sistema y el conocimiento del servicio alcanza al $(20,4 \%)$ de la población.

Finalmente, en cuanto a nuevos soportes para recibir información, los entrevistados muestran una inclinación clara a medios que empleen como soporte la plataforma de Facebook, pantallas luminosas, aplicativos para celulares y una canal en YouTube (figura 5). 


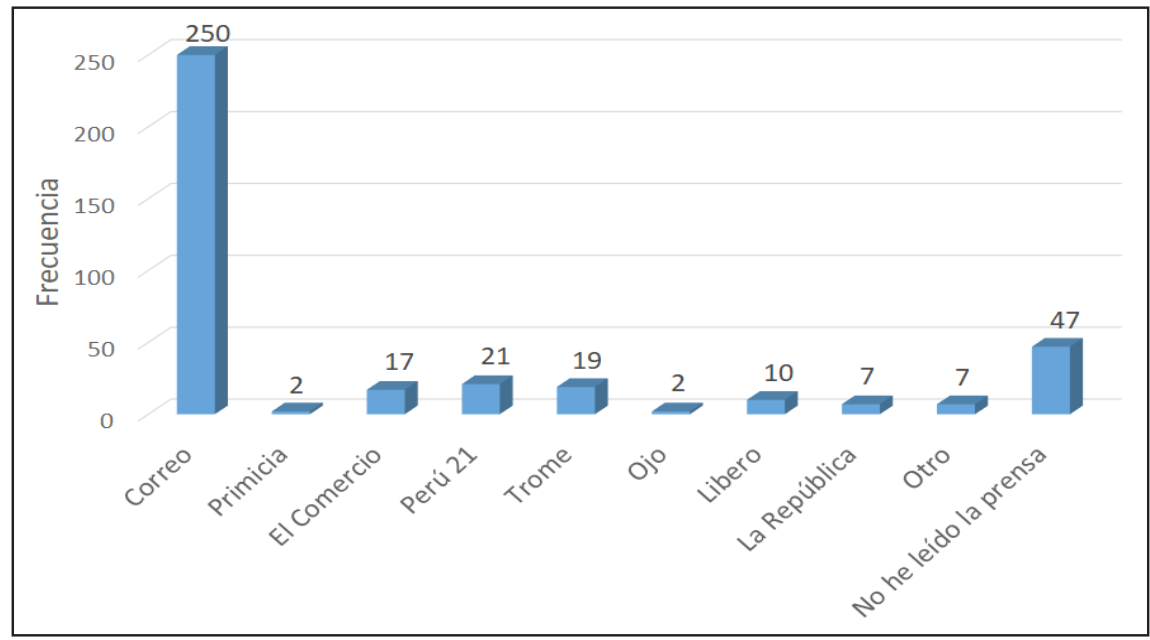

Figura $\mathrm{N}^{\circ} 2$, preferencia de diarios impresos por la población de estudio

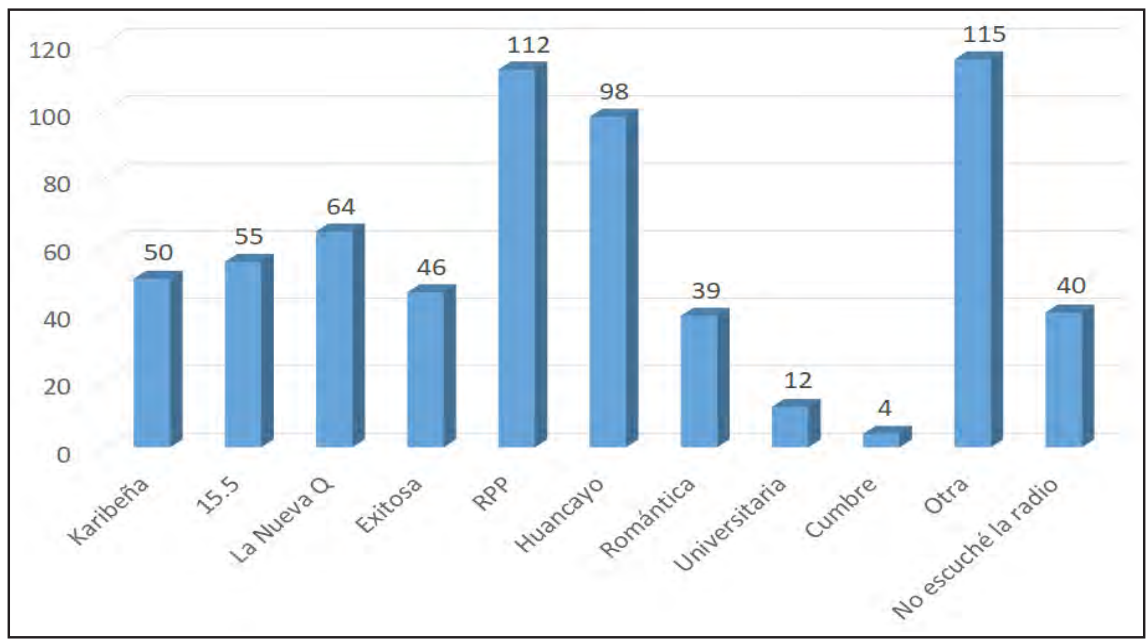

Figura $\mathrm{N}^{\circ} 3$, preferencia de emisoras radiales

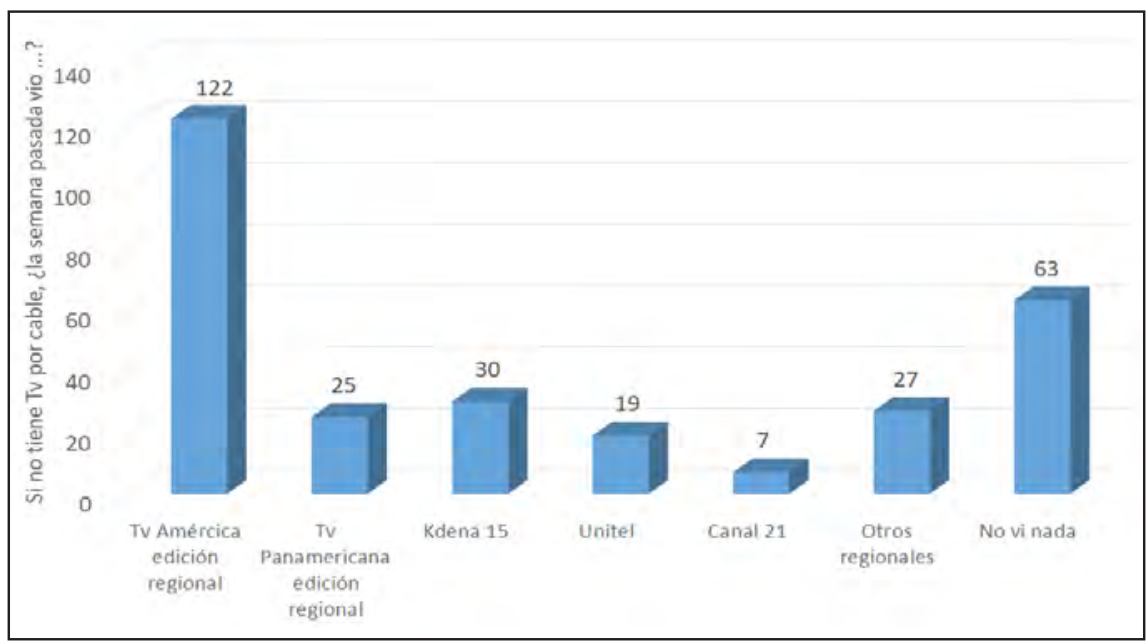

Figura $\mathrm{N}^{\circ} 4$, sintonía de canales de televisión, locales y nacionales 


\section{El contraste con la oferta de medios}

Para el análisis de la hipótesis planteada en relación a que los medios de comunicación de Huancayo carecen de contenido atractivo para la población de adultos de 25 a 35 años de Huancayo, se contabilizó el total de noticias difundidas del 01 al 08 de diciembre de 2015 y se contrastó esta oferta con la demanda hallada de temas en la presente investigación.

En la estadística se encontró que Correo presenta 29\% de noticias locales; $18 \%$ policiales; $17 \%$ regionales; $16 \%$ deportivas; $11 \%$ de política, entre otras (espectáculo, economía, salud, judiciales y educación). La información local es la gran ventaja del diario y por el valor noticioso de proximidad representa un gran argumento de venta.

En tanto que Primicia, muestra $24 \%$ de información antiguo", pese a contar con información más cercana a sus intereses, notas de salud, medicina y hogar que alcanzan el $11 \%$ del total del contenido.

En cuanto a la radio, para el estudio se analizó la programación de radio Huancayo y 15:50, en esta se usó la clasificación de Enma Rodero (2011), sobre tipo de programación, noticiosa y de entretenimiento, precisamente ambas estaciones cuentan con programas informativos en el bloque matutino de 6 a 9 de la mañana, posteriormente se orientan a un segmento de mujeres hasta el mediodía, en tanto que la programación por la tarde está más orientada a los adolescentes y jóvenes con programas de concurso.

En este caso, se observa que no hay contenidos específicos ligados a cobertura de noticias de interés que satisfagan al segmento objetivo, sin embargo, la radio es un medio con gran audiencia y

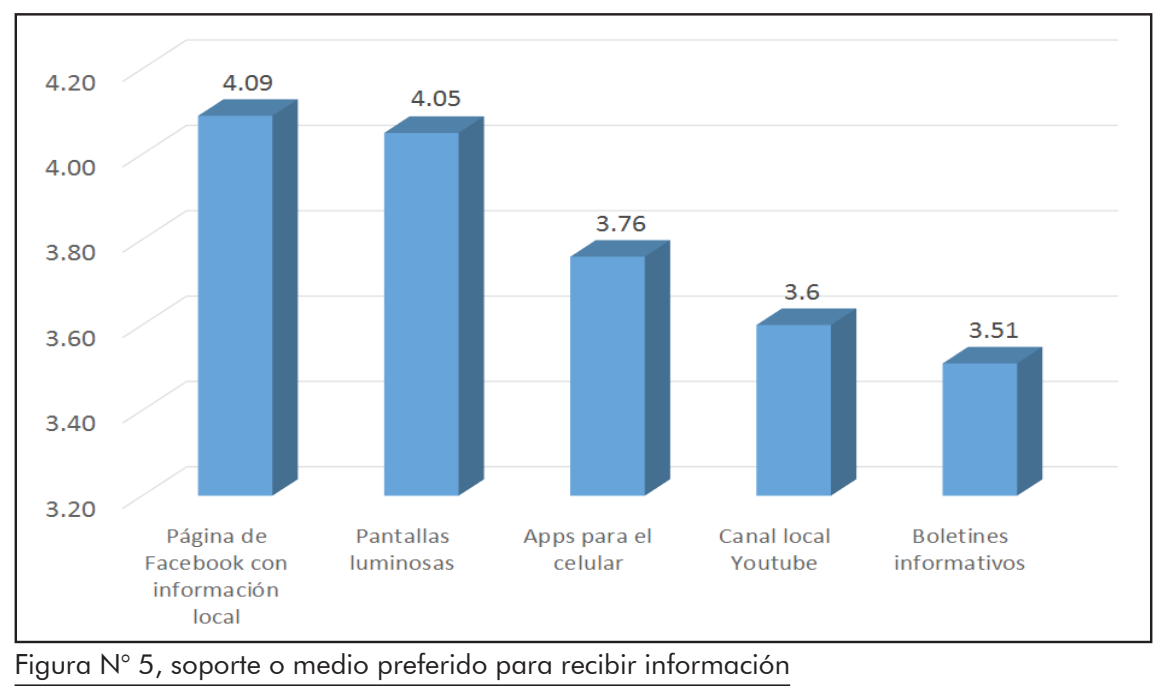

local, $19 \%$ de contenido ligado al sector educación, $11 \%$ a deportes, similar porcentaje asociado a salud, hogar y muestra solo 3,2 \% en cuanto a policiales y 5 $\%$ de política entre otros.

Al respecto, los grupos focales, especialmente el de varones, señalaron que asociaban a Correo con notas policiales ligadas a hechos de sangre, esto, pese a que en contenido hay más notas locales, esto puede asociarse a las portadas del diario del grupo El Comercio, debido a que prioriza sucesos impactantes, entre los titulares de esa semana se encuentran: "Racha de accidentes"; "Bombardeo en la selva" y "Va a fiesta y acaba en río".

Mientras que Primicia, tiene más noticias locales, así como del sector educación, no obstante, los participantes del grupo focal señalaron que por la edición, calidad y diseño les parecía un "diario credibilidad (Consejo Consultivo de Radio y Televisión [CONCORTV], 2015), esto se asocia a que no requiere de atención exclusiva, en este sentido implica que las personas suelen combinar quehaceres laborales y domésticos mientras escuchan la programación.

Para el caso televisivo, las señales de Cadena TV (ex Kdena 15) y Unitel, tienen programación local limitada, los espacios se concentran en noticieros matutinos y en el horario estelar (por la noche), así como programas dirigidos a la familia, uno ligado al panorama económico y otro hacia música folclórica.

Salvo por el programa de la Casa 15, de Cadena TV, no hay un programa que cuente con programación dirigida a la familia y con contenido de medicina, salud, educación, tecnología o medio ambiente. 


\section{DISCUSIÓN}

Al comparar las cifras del contenido noticioso de la prensa escrita frente a los temas de interés de la población de estudio vemos que la oferta satisface solo parcialmente y hay ausencias notorias como temas de medio ambiente, ciencia y tecnología, familia y educación, así como información deportiva de disciplinas distintas al fútbol.

Asimismo, la medición de audiencias se concentra en los indicadores de rating televisivo, sintonía radial y lectoría, que es importante pues mide la demanda, pero hace poco énfasis entre los temas específicos que requieren con mayor prioridad los pobladores de Huancayo.

CONCORTV (2015), indica que el consumo promedio entre lunes a viernes de TV es de tres horas con 12 minutos, radio, tres horas con 18 minutos e internet, dos horas con 54 minutos, además, la muestra analizada advierte como contenidos inapropiados en $\mathrm{TV}$, violencia (74\%), situaciones de mal ejemplo para la sociedad (64\%), discriminación (56\%), palabras groseras (54\%) entre otros, además en concordancia con lo hallado en el estudio se corrobora que la radio local tiene mayor aceptación (35\%), señala que la escucha a diario, frente a solo el $14 \%$ que ve televisión local.

Además, desde el punto de vista del marketing los medios de Huancayo aplican una filosofía de dirección centrada en la producción y el producto y en ciertos casos en la transición al marketing para descubrir las demandas de sus audiencias, puesto que se programa y produce aquello que se conoce, informativos $y$ noticias, pero aún no se identifica claramente o segmenta de forma adecuada a las audiencias, emitiendo programas genéricos que compiten sin diferenciación clara.

Sin ser apocalípticos, recordando el título de uno de los libros del desaparecido Umberto Eco, los medios afrontan un proceso de transición muy complejo que puede implicar la desaparición de algunos, por ello deben plantear estrategias que les permitan mantener audiencias sin rechazar las ventajas de los medios digitales y sociales a modo de combinar un lenguaje multimedia y de difusión de contenido a través de múltiples plataformas.

La alta penetración de los teléfonos inteligentes y el acceso a internet en las zonas urbanas, implica que se cambien soportes y se comience a desarrollar más productos como aplicativos para móviles, la tan reclamada página de noticias en Facebook, así como ofertas digitales, debido a que la lectura y seguimiento noticioso y de contenido por internet es muy escaso.

\section{REFERENCIAS BIBLIOGRÁFICAS}

Alfaro, R. (1997). Investigadores y comunicadores ¿dos orillas sin unir? La Pizarra (22).

Araujo, P. y Mayorga, D. (2002) Marketing estratégico en la empresa peruana. Lima, Perú:

Universidad del Pacífico.

Baran, S. e Hidalgo, J (2005). Comunicación masiva en Hispanoamérica. Cultura y literatura

mediática. México: Mc Graw Hill.

Biagi, S (2009). Impacto de los medios de comunicación. México: Cengage Learning.

Carrillo, S. (2015) ¿Qué libertad? ¿̇a de pensar? Medios, libertad de expresión y concentración de

medios en el Perú. Nuevos desafíos para la comunicación social en el Perú y en América Latina. Lima, Perú: Fondo Editorial de la UNMSM.

CONCORTV (2015). Estadísticas de la radio y la televisión en el Perú, disponible en

http://www.concortv.gob.pe/wp-content/ uploads/2015/03/Infografia-estad\%C3\%ADsticas2015-pdf.pdf

Dominick, J. (2006). La dinámica de la comunicación masiva. México: Mc Graw Hill.

Espinoza, J. (productor) (2015) Huancayo también marcha en contra de la "TV basura". Vídeo de

YouTube Disponible en https://www.youtube.com/ watch? $v=+91 \times w 8 S \_q Z Y \& t=87 \mathrm{~s}$

García, E. (2010) Investigación de mercados para pymes y empresas que recién comienzan. Lima,

Perú: Universidad del Pacífico.

Jarvis, J. (2010). Y Google, ¿̇cómo lo haría? Colombia: Planeta.

Jensen, K. (2014). La comunicación y los medios. Metodologías de investigación cualitativa y

cuantitativa. México: FCE.

Kotler, P., y Armstrong, G. (2013). Fundamentos de marketing. México, México: Pearson Educación.

Montesinos, J. (2008) Cómo aplicar el marketing en el Perú. Lima, Perú: Color \& trazos.

Rodero, E. (2011). Creación de programas de radio. Madrid, España: Síntesis. 